\title{
Code Clones in Feature-Oriented Software Product Lines
}

\author{
Sandro Schulze \\ University of Magdeburg, Germany \\ sanschul@iti.cs.uni-magdeburg.de
}

\author{
Sven Apel \\ University of Passau, Germany \\ apel@uni-passau.de
}

\author{
Christian Kästner \\ University of Magdeburg, Germany \\ kaestner@iti.cs.uni-magdeburg.de
}

\begin{abstract}
Some limitations of object-oriented mechanisms are known to cause code clones (e.g., extension using inheritance). Novel programming paradigms such as feature-oriented programming (FOP) aim at alleviating these limitations. However, it is an open issue whether FOP is really able to avoid code clones or whether it even facilitates (FOP-related) clones. To address this issue, we conduct an empirical analysis on ten feature-oriented software product lines with respect to code cloning. We found that there is a considerable amount of clones in feature-oriented software product lines and that a large fraction of these clones is FOP-related (i.e., caused by limitations of feature-oriented mechanisms). Based on our results, we initiate a discussion on the reasons for FOP-related clones and on how to cope with them. We exemplary show how such clones can be removed by the application of refactoring.
\end{abstract}

Categories and Subject Descriptors $\quad$ D.2.3 [SOFTWARE ENGINEERING]: Coding Tools and Techniques

General Terms Design, Languages

Keywords Code clones, software product lines, feature-oriented programming, refactoring

\section{Introduction}

Code cloning, that is, the replication of code fragments in source code, is known to be a serious and common problem in objectoriented programming $(O O P)[6,10,35]$. Numerous studies have shown that code clones occur frequently in software systems, ranging from $7 \%$ to $23 \%[6,10,21,26,32]$. Although recent studies discuss the harmfulness of code cloning controversial, it is widely accepted that code clones have a negative effect on the software system, in terms of a decrease of maintainability and the introduction of errors [10, 23, 35]. The causes for code cloning have been widely discussed and include, amongst others, shortcomings of the host language, which force the programmer to introduce code clones without any alternative.

Recently, novel programming paradigms such as feature-oriented programming $(F O P)$ gained attention that aim at overcoming certain limitations of OOP. In the context of FOP, a feature is an increment in program functionality. The functionality encompassed by a feature is encapsulated in a cohesive unit called feature module. Feature modules can be used to build a software product line (SPL)

Permission to make digital or hard copies of all or part of this work for personal or classroom use is granted without fee provided that copies are not made or distributed for profit or commercial advantage and that copies bear this notice and the full citation on the first page. To copy otherwise, to republish, to post on servers or to redistribute to lists, requires prior specific permission and/or a fee.

GPCE'10, October 10-13, 2010, Eindhoven, The Netherlands.

Copyright (C) 2010 ACM 978-1-4503-0154-1/10/10 .. \$10.00 by means of compositional programming. Typically, an SPL consists of a set of features ${ }^{1}$. A user-defined subset of these features is composed to synthesize a tailored product. In comparison to OOP, FOP aims at a higher modularity and reusability. However, up to now, no studies have been conducted to explore the presence and effects of code clones in feature-oriented software product lines. Based on our experience with SPL development, we assume that, although FOP has the potential to avoid OOP-related code clones, it may introduce FOP-related clones.

We want to shed light on the issue of code cloning in FOP and raise a number of fundamental questions: Do code clones exist in feature-oriented SPLs? Is FOP prone to introduce FOPrelated clones especially in the context of SPLs? If clones exist, is the development process of the SPL crucial (e.g., from scratch or by refactoring legacy applications)? And finally, what can we do against code clones in feature-oriented SPLs?

To answer these questions, we perform a detailed code clone analysis of ten feature-oriented SPLs, six implemented from scratch and four refactored from legacy applications. We use the established clone detection tool CCFinder ${ }^{2}$ [24] to obtain the code clones for the respective product lines. In particular, we make the following contributions:

- We perform a conceptual analysis of code clones in FOP. We identify limitations of FOP that are likely to introduce code clones. For example, the coarse-grained granularity of extensions in FOP is a limitation that may lead to code clones.

- By means of a case study on ten different non-trivial featureoriented SPLs, we analyze the amount and characteristics of clones in FOP.

- We explore and discuss whether code clones occur independently of the fact that an SPL has been developed from scratch or refactored from a legacy application.

- Based on the results of our analysis, we discuss possibilities of removing clones by means of refactoring without breaking the validity of the underlying SPL.

- We initiate a discussion on the existence and effect of code clones in FOP.

Based on our analyses, we will partly answer the questions raised above. In a nutshell, we gained the following insights:

- A considerable amount of (FOP-related) code clones actually exists in the analyzed SPLs.

- Code clones can be removed (in parts) through the application of refactorings that are tailored to feature-oriented SPLs.

\footnotetext{
${ }^{1}$ The terms feature and feature module are used synonymous for the remaining paper, because there is a 1:1 mapping between problem and solution space

${ }^{2} \mathrm{http}: / /$ www.ccfinder.net
} 


\section{Background}

\subsection{Code Clones}

We give a short overview of research on code clones to lay the foundation for the remaining sections. Code clones are known to have a negative effect on programs. Amongst others, increased maintenance costs and an increased probability of bugs are the most devastating consequences [37, 43].

Code clones are classified into four categories: type-I (identical), type-II (similar), type-III (statements are removed, added or changed) and type-IV (semantically similar) clones. In Figure 1, we give an example for a type-II clone pair, which we detected in one of our case studies, the graph product line (GPL) [34]. The two code fragments differ only in the variable names urep and vrep (Lines 4-8 and Lines 10-15). Furthermore, corresponding code clones can be encompassed to clone classes, e.g., by establishing an equivalence relation between them. Such clone classes are useful for further steps such as analysis or removal since they allow to treat the contained clones as a unit. Several approaches for the detection of clones exist, which are text-based [6, 17], tokenbased [24, 32], tree-based [10, 31] and metric-based [30, 35] clone detection. For detailed information on clone detection techniques and tools we refer to Roy et al. [44].

The result of the clone detection can be used for further processing. Generally, two approaches exist: code clone removal and code clone management, of which the latter implies that the clones remain in the program. For code clone removal, on which we concentrate in this paper, an analysis of the detected clones is carried out to obtain information on the clones useful for their removal, e.g., identifying corresponding clones (clone classes) or determining the clone type. Then, clones are removed using refactorings that is restructuring the program in a behaviour-preserving way [18]. Several approaches exist, in which different refactorings are applied manually or (semi-)automatically to remove clones [7, 19].

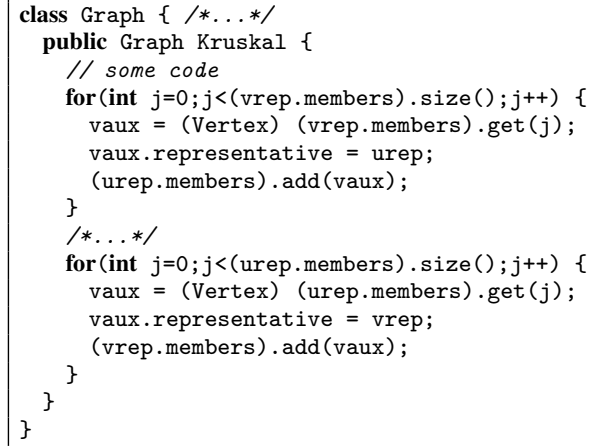

Figure 1. An example of a type-II code clone, taken from graph product line (GPL) [34]

\subsection{Feature-Oriented Software Product Lines}

An SPL is a set of software-intensive systems that share a common, managed set of features [13]. In the context of this paper, a feature is an increment in functionality that implements a stakeholder's requirement $[9,42]$. To create a concrete program from an SPL, the user selects the desired features, typically a subset of all features of the SPL, and a generator generates the tailored program (a.k.a. variant). Typically, different programs of an SPL contain common as well as different features.

The variability of an SPL is described by a feature model [25]. A feature model defines the features of an SPL and their dependencies. In Figure 2, we depict a feature model of a Stack prod-
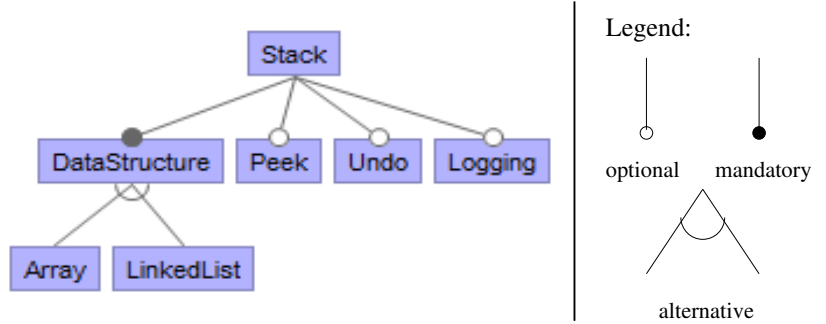

Figure 2. Feature model of the Stack SPL

uct line. The root of the model represents the SPL itself whereas the remaining nodes represent the individual features. Features can be optional or mandatory and beyond that, these features can be grouped as alternative features. Optional and alternative features are used to express variability in an SPL while mandatory features express commonalities across all variants of the SPL.

There are different approaches to implement SPLs, e.g., frameworks [22] or conditional compilation [27, 41]. Here, we use FOP, a language-based approach (a.k.a compositional approach) that gained much attention in the past and aims at the modularization of a program by decomposing it along features. Several FOP languages and tools exist, such as AHEAD [9], FeatureHouse [5] or FeatureC++ [3], which provide various mechanisms for the modularization and composition of features. The core idea is that the functionality encompassed by a feature is implemented by a cohesive unit called feature module. As a result, we obtain a clean relationship between the feature and its implementation unit. The increment of functionality, introduced by a feature, is realized by adding new structures such as classes or methods and refining existing ones, such as extending a method.

\begin{tabular}{|c|c|}
\hline Feature Stack & Feature Undo \\
\hline $\begin{array}{l}\text { class Stack }\{\ldots \\
\text { void } \operatorname{push}(\text { int } v)\{/ * \ldots * /\} \\
\text { int } \operatorname{pop}()\{/ * \ldots * /\}\end{array}$ & \multirow{4}{*}{$\begin{array}{l}\text { refines class Stack }\{\ldots \\
\text { int backupPush; } \\
\text { void undo }()\{/ * \ldots *\} \\
\text { void push }(\text { int } \mathrm{v})\{ \\
\text { backupPush=v; } \\
\text { original }(\mathrm{v}) ; \\
\}\end{array}$} \\
\hline Feature Peek & \\
\hline $\begin{array}{l}\text { refines class Stack }\{ \\
\quad \text { int } \operatorname{peek}()\{/ * \ldots *\}\end{array}$ & \\
\hline & \\
\hline
\end{tabular}

Figure 3. Feature-oriented implementation of Stack with features Peek and Undo

In Figure 3, we show three features of our Stack product line. Feature Stack is the basic feature of our product line, in which the class Stack is initially declared. The two features Peek and Undo refine this class in order to add functionality (indicated by 'refines class...'). Feature Peek introduces a new method that enables the user to copy the upmost element of the stack. Feature Undo introduces a method undo and extends the existing method push by an additional statement at the beginning so that the upmost element is assigned to a backup variable. The keyword original invokes the method push of the original class Stack. We use the ' ' '-operator to denote the composition of features to a program. Although in our example each feature contains only one class, typically multiple classes belong to a feature.

\section{Reasoning about Code Clones in Feature-Oriented SPLs}

Limitations of (object-oriented) programming languages are discussed as a reason for code clones [8, 43]. Mechanisms such as 


\begin{tabular}{|c|c|c|c|c|c|c|c|c|c|c|c|c|c|}
\hline & \multicolumn{2}{|c|}{ (a) clone detection } & \multicolumn{7}{|c|}{ (b) syntactical classification } & \multicolumn{4}{|c|}{ (c) feature-related classification } \\
\hline & $\mathrm{SLOC}_{\text {clones }}$ & $\mathrm{CR}_{\mathrm{cd}}$ in $\%$ & IS & FS & WS & DS & MD & $\mathrm{TD}$ & $\mathrm{CR}_{\mathrm{sc}}$ in $\%$ & CPF/A & $\mathrm{CDF}$ & $\mathrm{SLOC}_{\text {clones }}$ & $\mathrm{CR}_{\mathrm{FOP}}$ in $\%$ \\
\hline GPL & 731 & 37 & 0 & 14 & 0 & 0 & 480 & 185 & 35 & $652 / 652$ & 0 & 652 & 34 \\
\hline GUIDSL & 900 & 7 & 20 & 0 & 24 & 0 & 403 & 443 & 7 & $98 / 98$ & 0 & 98 & 1 \\
\hline Notepad & 291 & 28 & 130 & 0 & 0 & 0 & 18 & 58 & 20 & $130 / 0$ & 0 & 130 & 12 \\
\hline PKJab & 203 & 6 & 18 & 12 & 0 & 0 & 0 & 90 & 3 & $0 / 0$ & 0 & 0 & 0 \\
\hline TankWar & 1000 & 20 & 132 & 4 & 0 & 0 & 371 & 242 & 15 & $662 / 634$ & 0 & 680 & 13 \\
\hline EPL & 18 & 12 & 0 & 0 & 0 & 0 & 0 & 18 & 12 & $18 / 18$ & 0 & 18 & 12 \\
\hline BerkeleyDB & 952 & 2 & 65 & 0 & 0 & 0 & 436 & 207 & 1.5 & $69 / 69$ & 67 & 357 & $<1$ \\
\hline MobileMedia & 716 & 16 & 24 & 0 & 0 & 0 & 46 & 482 & 13 & $16 / 16$ & 135 & 305 & 7 \\
\hline Violet & 784 & 11 & 56 & 24 & 0 & 0 & 30 & 315 & 6 & $220 / 162$ & 108 & 328 & 5 \\
\hline Prevayler & 131 & 2 & 6 & 0 & 0 & 0 & 62 & 58 & 2 & $0 / 0$ & 0 & 16 & $<1$ \\
\hline
\end{tabular}

CR: clone ratio; IS: IfStatement; FS: ForStatement; WS: WhileStatement; DS: DoStatement; MD: MethodDeclaration; TD: TypeDeclaration; CPF/A: clones with common parent feature/portion of clones from alternative features; CDF: clones with common dependency feature;

Table 2. Statistics of clone detection and analysis

refactoring opportunities for code clone removal, for instance, by applying Extract Method or Pull Up Method refactorings, tailored to SPLs [18]. All clone classes with different syntactical categories are filtered out and not considered for further analysis.

We list the results of this classification in Table 2 (b). For each category, we give the amount of clones (SLOC), and in the last column we provide the clone ratio for each of the considered SPLs (column $\mathrm{CR}_{\mathrm{sc}}$ ). For instance, the TankWar product line has the following amount of code clones: 371 SLOC related to method declarations, 242 SLOC related to type declarations, and 132 SLOC related to if statements. Overall, this product line has a clone ratio of $15 \%$, i.e., there are some clones that are not related to the considered syntactical categories.

Feature-related classification. In the second step of our analysis, we identify the nature of code clones, i.e., whether they are FOPrelated or not. For determining the nature we have to consider corresponding code clones as a whole. Thus, we analyzed the clone classes created after clone detection and selected for further analysis by syntactical classification. First of all, we define the following condition: A clone class is FOP-related, if its member clones affect at least two features, i.e., the corresponding clones must occur in at least two different features. Code clones of a clone class that affect only one feature, occur within one class or between different classes of a single feature. Since this kind of cloning happens in a usual OOP program as well, we assume that these clone classes are OOP-related. These clones are relevant too, but outside the scope of this paper.

After the classification, we analyzed the FOP-related clones to gain information on the causes of cloning as well as to identify possible refactorings applicable for code clone removal. In detail, we identified dependencies amongst features that share common code. We discuss the concrete correlation between feature dependencies, obtained by this analysis step, and refactoring in Section 5 .

We show the result of the whole second analysis step in Table 2 (c). The respective columns contain the results of our analysis. In column $\mathrm{SLOC}_{\text {clones, }}$ we list the total amount of clones between multiple features. Column $C P F / A$ indicates the number of clones that occur in features with a common, direct parent feature and beyond that, how many of these clones occur in alternative features. Furthermore, we list how many clones (using the SLOC metric) occur in dependent features (column $C D F$ ). We describe such dependencies in Section 5 in detail. Finally, we list the clone ratio in column $\mathrm{CR}_{\mathrm{FOP}}$. For instance, the already mentioned TankWar SPL contains 680 lines of FOP-related code clones (column $\mathrm{SLOC}_{\text {clones }}$ in Table 2), which are $13 \%$ compared to the whole code size (column $\mathrm{CR}_{\mathrm{FOP}}$ ). The first part of column $C P F / A$ indicates that 662 lines of code clones exist in features with a common, direct parent feature $(C P F)$. The second part of this column $(A)$ indicates that 634 lines of code clones exist in alternative features. In the following subsection we will have a closer look on the results.

\subsection{Results}

During our analysis, we collected various data. Here, we only describe our results from in Table 2. For a discussion and interpretation of the results refer to Section 4.4. We structure our description according to our analysis steps and examine the differences that may result from the different development process of our SPLs.

The clone ratio, given at several points in this subsection, is always related to the total amount of code (SLOC) for each SPL. Additionally, we calculated the percentage of the average and the standard deviation $(a \pm s)$ on the clone ratio of all considered SPLs. Next, we state the results for our three analysis steps, that is, clone detection, syntactical classification and feature-related classification. Additionally, we put our focus on how the results depend on the development process of the analyzed SPLs.

Amount of code clones. The results of our initial clone detection reveal that there is a significant amount of clones in feature-oriented SPLs (cf. Table 2 (a)). Regarding all considered SPLs, $15 \pm 10 \%$ of the overall code are clones. We observed considerable differences regarding the clone ratio of the particular SPLs that ranges from $2 \%$ to $37 \%$, which is also reflected by the relatively high standard deviation. Beyond this, we noticed that two of the smallest SPLs (GPL and Notepad) exhibit the highest clone ratio values with $37 \%$ and $28 \%$ respectively. By contrast, the two largest SPLs (Berkely $D B$ and GUIDSL) are amongst those with the lowest clone ratio value.

Refactorable clones. With our first analysis step, we aimed at detecting clones that could be target to refactorings because of their syntactical characteristics. The data (cf. Table 2 (b)) reveal that there is still a huge amount of clones that may be removable indicated by a total amount of code clones of $12 \pm 9 \%$. In addition, we observed the clone ratio decreases in comparison to the initial clone detection in almost all SPLs. We observed that the clone classes, filtered out by the syntactical classification, mainly fall into the three categories IfStatement, MethodDeclaration, and TypeDeclaration. Particularly, we noticed the high amount of code clones in category TypeDeclaration, which means that whole classes has been cloned.

FOP-related clones. The data resulting from the last analysis step (cf. Table 2 (c)), reveal, that there are code clones that are 
FOP-related by our definition. Nevertheless, we observed that four feature-oriented SPLs (GUIDSL, PKJab, Berkeley DB, and Prevayler) contain (almost) no FOP-related clones. We assume that this results from the fact, that these SPLs exhibit the lowest clone ratio even in the initial clone detection (cf. Table 2 (a)) and that the existing clones are OOP-related. Generally, we observed that the clone ratio is considerable lower than the clone ratio after syntactical classification for all SPLs except of GPL. Regarding all SPLs, the amount of clones is $9 \pm 9 \%$, which reveal that there is a high diversity between the clone ratio of the several SPLs. Actually, four SPLs exhibit a clone ratio greater than $10 \%$ whereas the clone ratio of the remaining SPLs is less than $8 \%$. Finally, we observed that the FOP-related clones are mostly distributed over alternative features that additionally have a common parent feature (column CPF/A in Table 2 (c)). Only in three SPLs (MobileMedia, BerkeleyDB, and Violet), clones are contained in features that have a common dependency feature instead of a common parent.

From scratch vs. Decomposed. Considering all of the data we collected, we observed one peculiarity: Throughout all analysis steps, the amount of clones in SPLs developed from scratch is significant higher than in SPLs decomposed from legacy applications. This is also indicated by the amount of clones that is significantly higher for SPLs from scratch ( $19 \pm 12 \%$ in Table 2 (a), $12 \pm 12 \%$ in Table 2 (c)) than for SPLs from legacy applications (10 $\pm 5 \%$ in Table 2 (a), $5 \pm 4 \%$ in Table 2 (c)). The SPLs decomposed from legacy applications were developed originally object-oriented and finally, were decomposed manually or automatically [28]. Beyond that, the data reveal that the diversity of clone ratios between the individual SPLs from scratch is very high, which indicates that there are even differences amongst those SPLs. Finally, we observed that both kinds of SPLs contain FOP-related as well as OOP-related clones.

\subsection{Discussion}

Next, we discuss the results of our case study along with the research questions, raised in the introductory section.

Do code clones exist in feature-oriented SPLs? Based on the results of our analysis, we conclude that a considerable amount of code clones actually exist in feature-oriented SPLs. Beyond that, we observed that there are significant differences, regarding the amount of clones between the analyzed SPLs in general, and in a few of them the amount is even negligible. In addition, some of the smallest SPLs exhibit the highest amount of clones. However, considering the overall result of the clone detection, we can not discover a correlation between SLOC metric and clone ratio.

Is FOP prone to introduce FOP-related clones in SPLs? Our results indicate that there are FOP-related clones in the analyzed SPLs. An interesting observation is that the majority of these FOPrelated clones occur between alternative features. This observation coincides with the limitations of FOP analyzed by us and other researchers before. But this observation indicates that there is a high potential for code clone removal, because we can possibly extract the respective clones into a common feature (cf. Section 5). By contrast, we also detected clones that are FOP-related by our definition, which do not occur in alternative features. Considering our data, we can not clearly infer why these clones occur. But even without these clones it is a matter of fact that feature-oriented SPLs contain FOP-related clones.

Is the development process of the SPL crucial for code cloning? Our results show differences between the analyzed SPLs that can be ascribed to the development process (from scratch vs. decomposed). In detail, the SPLs developed from scratch contain a significant higher amount of clones than the SPLs decomposed from legacy applications. Considering our data, we observe a relation between FOP-related clones and alternative features. Indeed, the SPLs from scratch have a considerable amount of alternative features while the decomposed SPLs have not. We conclude that a) alternative features especially lead to code clones and b) the SPLs from scratch contain a higher amount of this kind of features. This observation is supported by the fact that the SPLs decomposed from legacy applications were not designed with variability in mind and thus contained no or only few alternatives before decomposition. As a result, the variability was mostly introduced by optional features during the decomposition process.

Another reason may be that the programmers of the SPLs from scratch were not capable to exploit all concepts and mechanisms of FOP (as often observed with new programming paradigms). Hence, they may have introduced clones unnecessarily or missed to factor out clones where it was possible with the mechanisms of FOP.

How to deal with clones in feature-oriented SPLs? During our analysis, we particularly looked at the refactoring potential of the detected clones. The corresponding data reveal that a large portion of the overall detected clones exhibits characteristics that indicate refactoring opportunities. One interesting observation we made is that a huge amount of clones between alternative features are across method declarations. These clones can be refactored by pulling them up to the common, direct parent feature. We will have a closer look to concrete refactorings in Section 5.

However, our data do not reveal information on the concrete amount of actual refactorable clones. For instance, two cloned methods may be similar except of one statement, that differs in the call to another method as in Figure 5. As a result, it will be hard to apply a refactoring such as Pull Up Method to these clones. This requires a more detailed analysis, which we present exemplarily for one of the considered SPLs in Section 5. Besides refactoring, other possibilities exist for managing clones, we did not consider in our analysis, such as clone tracking [16] or linked editing [46]. The idea of both approach is that the detected clones remain in the code but information of their existence is used for their management, e.g., for changing code clones simultaneously. Apart from that, we believe that code clone removal, if applicable, is the most suitable practice for dealing with clones.

\subsection{Threats to validity.}

Single FOP language. Although FOP is a general paradigm, it depends to some extent on the mechanisms of the underlying language. As a result, different FOP languages exist (e.g., for $\mathrm{C}++$ and Java) that may lead to different implementations for featureoriented SPLs. In this paper, we focused only on FOP languages based on Java so that the results of our analysis are comparable. However, the classification we made along with our analysis is also valid for other languages in theory, e.g., $\mathrm{C}++$ or $\mathrm{C \#}$. Although no empirical evaluation for other languages yet exists, we assume that our analysis is independent of the underlying language.

Selected SPLs. A major problem with case studies is that the selected programs may be biased an thus the results are meaningless overall. In addition, only few SPLs exist that are implemented using FOP (based on Java) which might reduce the significance of our study. To address this problem, we considered all available feature-oriented SPLs for our study. Beyond that, the selected SPLs are from different domains and of different size. Nevertheless, one problem remains, that is, all of the analyzed SPLs are prototypical implementations from academia. Hence, there is a lack of comparable results of SPLs with industrial strength, which is also caused by the fact that such systems not exist for FOP. Nevertheless, the considered SPLs have been implemented by different authors and for other purposes than analyzing them for code clones. Consid- 
ering the SPLs decomposed from legacy applications it is worth to mention that these SPLs where decomposed without code clone in mind. Hence, we can definitively exclude that the results of our empirical analysis are biased because of code clone awareness.

Classification of FOP-related clones. During our analysis, we proposed a classification for FOP-related clones based on the relation of the affected features. However, we detected clones for which we can neither infer why these clones occur nor if they are FOPrelated indeed. One possibility is that these clones are contained in features that implement homogeneous crosscutting concerns. Since this kind of concerns occurs in OOP programs as well, the respective clones may be not purely FOP-related. Beyond that, our condition for FOP-related clones is rather a criteria that can be used to omit clones that are clearly OOP-related. As a result, this condition can not ensure that FOP-related clones are caused by FOP exclusively. Hence, we should refine this condition to be more restrictive in our classification of what an FOP-related clone is. However, we defined a lower bound with our definition of what an FOP-related code clone is, which can be used as a base for future work.

\section{Removal of (FOP-related) Code Clones}

Along with our empirical analysis, which we presented in Section 4 , we focused on refactoring opportunities for removing the detected (FOP-related) clones. In this section, we discuss how the analysis results can be used for deriving concrete refactorings. Subsequently, we apply these refactorings exemplarily to one of our case studies, the TankWar product line.

\subsection{Extracting Code Clones from Features}

To remove FOP-related clone classes there are different possibilities. A general pattern is that we want to replace the replicated code in multiple locations by a single reusable code fragment.

For example, in the simple case that an SPL always requires one of two alternative features, and both features introduce the same method, then we can remove all cloned instances of the method and introduce it only once in the root feature. With this modification, we eliminate cloning and the method is always available from the root feature. Obviously, we cannot move every cloned code fragment into the root feature. If it is valid to select none of the features containing cloned code, moving code to the root feature would bloat the code base of variants in that none of these features is selected. Additionally, it can be considered as violation of separation of concerns.

A general solution is to move cloned code into a newly created feature that is selected if and only if at least one of the features containing cloned code is selected. Consider the feature model in Figure 7 (a) and assume that some code between features C and D is cloned. In this case, we could create a new parent feature $\mathrm{X}$ for $\mathrm{C}$ and $\mathrm{D}$ and move the cloned code there as illustrated in Figure 7 (b). Alternatively, we can create a new feature X somewhere else in the feature model and use a cross-tree constraint ( $\mathrm{X}$ equals $\mathrm{C}$ or D) to enforce the previous semantics as in Figure 7 (c). Of course, we can also search the feature model for existing features that would meet the condition, instead of creating a new one. Note that both transformations of the feature model preserve all existing variants and do not create new variants (called feature model refactoring) [45].

The pattern of moving cloned code to a single new location works uniformly for different kind of clones: cloned types, cloned methods... etc.

\subsection{Exemplary Refactoring of FOP-related Code Clones}

To evaluate the applicability of refactorings to FOP-related clones, we performed code clone removal by manually applying refac-

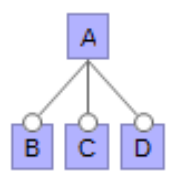

(a) Original

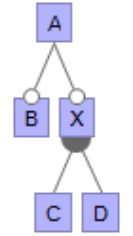

(b) Alternative \#1

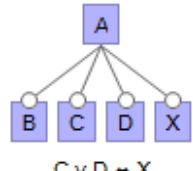

$C \vee D \Leftrightarrow X$

(c) Alternative \#2
Figure 7. Feature model edits for code clone removal

torings for one of our case studies, called TankWar. TankWar is a shoot 'em up game, running on PC and handy, that was developed by students of the University of Magdeburg. We selected this product line, because it has both, high clone ratio and relatively high amount of FOP-related clones, and it is of medium size (approx. 5000 SLOC). The game was developed as product line because it must adhere to strong portability requirements [1]. For instance, TankWar has been developed for PC and handy, which have different constraints regarding memory or display. Even between handys, there can be considerable differences, e.g., a modern smartphone has more memory than a five year old handy. As a result, the developer must be able to tailor the game in order to achieve the best game quality. In Figure 6, we show the feature model of the TankWar product line where features such as Image and Sound are specific for different platforms. Furthermore, we can see several alternative feature groups and according to our analysis (cf. Table 2 (c)), these features contain a large portion of code clones. Hence, we expected that removing these clones by refactoring is very promising.

Following our analysis results, fourteen clone classes emerged that contain potentially refactorable clones. We list these clone classes in Table 3, together with their syntactical category $(S C)$, the features containing the code clones $(C F)$, the target feature for the refactorings $(R F)$, and the applied refactorings (if possible). After a first review of these clone classes, we declared four clone classes (\#11 - \#14) as "not refactorable" for the following reasons: Three of them (\#11 - \#13), contained in different features below the feature Tools, consist of type-II clones which means that refactoring would be only possible with some workarounds. Since this leads to complicated code and, in this special case, to increased code size, we excluded these clone classes from the refactoring process. The fourth clone clone class (\#14) consist of type-III clones with notable differences so that a refactoring was not applicable. Beyond this, the clones are scattered over features that have neither a common parent nor other dependencies that are essential for the application of refactorings.

For the remaining ten clone classes that where finally subject of our refactoring process, we made the following initial observations. Obviously, most of the clones exist between alternative features that separate platform-dependent functionality. In addition, the clone classes fall only into three different syntactical categories (IfStatement, MethodDeclaration, TypeDeclaration), which coincides with our observation in Section 4 that almost all clone classes fall into one of these categories. Furthermore, we made some observations, which we not listed in the table due to space restrictions. First, all member clones, i.e., clones of a single clone class, have a common, direct parent feature. Second, clone classes with syntactical category TypeDeclaration (TD) in fact contained replicated methods or constructors as code clones. Hence, we treat them like clone classes of category MethodDeclaration (MD) for the refactoring process. Third, we observed that seven clone classes consist of type-I clones and three of type-II clones. 


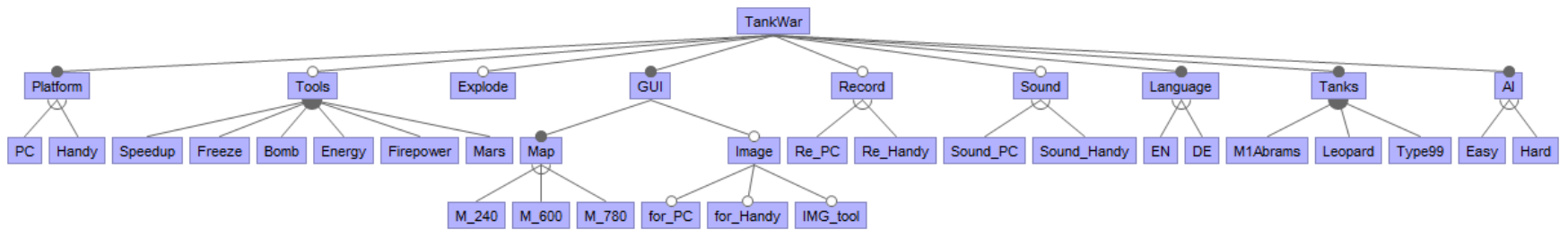

Figure 6. Feature model of the TankWar product line

\begin{tabular}{rllll}
\hline CC & SC & CF & RF & Refactorings \\
\hline$\# 1$ & TD & Leopard, Abrahams,... & Tanks & EM, PUM \\
$\# 2$ & MD & PC, Handy & Platform & PUM \\
$\# 3$ & IS & PC, Handy & Platform & EM, PUM \\
$\# 4$ & MD & PC, Handy & Platform & PUM \\
$\# 5$ & MD & PC, Handy & Platform & PUM \\
$\# 6$ & MD & PC, Handy & Platform & PUM \\
$\# 7$ & TD & PC, Handy & Platform & PUC \\
$\# 8$ & MD & Re_PC, Re_Handy & Record & PUM \\
$\# 9$ & TD & Re_PC, Re_Handy & Record & PUC \\
$\# 10$ & IS & TankWar, Tools & TankWar & EM \\
$\# 11$ & TD & Bomb, Freeze, ... & - & - \\
$\# 12$ & TD & Bomb, Freeze,... & - & - \\
$\# 13$ & TD & Bomb, Freeze, ... & - & - \\
$\# 14$ & IS & Handy, Re_Handy & - & - \\
\hline
\end{tabular}

CC: clone class; SC: syntactical category; $\mathrm{CF}$ : feature(s), containing the clones; RF: feature, the clones are refactored to; EM: Extract Method refactoring; PUM: Pull Up Method refactoring; PUC: Pull Up Constructor Body refactoring

Table 3. Overview of clone classes removed by refactorings

For the actual refactoring process, we had to tailor certain object-oriented refactorings to FOP. We call such refactorings feature-oriented refactorings ${ }^{7}$. For instance, we tailored the Pull Up Method refactoring so that it refers to features instead of classes. As a result, the application of this refactoring in the context of feature-oriented SPLs means that the respective method is moved from the current feature to its parent feature. In the same way, we tailored the Pull Up Constructor Body. In contrast, we used the Extract Method refactoring in its original form, because their is no difference between extracting a method in OOP and FOP.

During the application of the refactorings mentioned above, we made the following observations. Initially, we could apply the refactorings to all of the ten clone classes and consequently, remove the code clones. For three clone classes, we had to apply the Extract Method refactoring in advance, either for extracting the identical part of the clones (\#1) or for extracting if statements into methods (\#3, \#10). For two clone classes (\#7, \#9) we had to replace a value by a variable, which we initialized for each of the clones separately. Finally, we applied the (feature-oriented) Pull Up Method refactoring to all clone classes to remove the clones.

After the code clone removal process, we analyzed the TankWar product line again, according to the methodology introduced in Section 4. This lead to the following results. The amount of code clones has been decreased throughout all analysis steps. Regarding the initial clone detection, the amount of clones decreased from $20 \%$ (cf. Table 2 (a)) to $12 \%$. For the syntactical classification, the amount of code clones is $50 \%$ lower in the refactored SPL (7 \%) compared to the original one (15\%). Finally, we achieved a vast

\footnotetext{
${ }^{7}$ Note that this term is used in analogy to aspect-oriented refactorings [38] and different from the term used by Liu et al. [33], where it describes the process of decomposing (object-oriented) programs into features.
}

decrease of the amount of FOP-related clones. In the refactored SPL, only $4 \%$ FOP-related clones exist, which is three times lower than in the original SPL $(12 \%)$. We conclude that code clone removal through refactorings is a promising approach to remove FOP-related clones from feature-oriented SPLs.

\subsection{Discussion}

In the following, we shortly discuss the results mentioned above.

Firstly, we state that a large portion of FOP-related clones can be removed by refactoring. This fact may raise the question whether these clones occur due to copy\&paste activities rather than limitations of FOP. But even in this case it is unclear why the programmer copied the code. For instance, it is possible that the programmer was not aware of proper (feature-oriented) refactorings or mechanisms that can avoid the resulting code clones. As a result, these clones can be considered FOP-related to some extent as well.

Secondly, we observed that there are some limitations for the removal of FOP-related clones. On the one hand, all of the removed clones occurred in features with a common, direct parent feature and most of them were alternative features. Hence, we can make no clear statement on removing clones caused by fine-grained extensions or crosscutting concerns. On the other hand, the detected clones where mostly identical (type-I) or had only slight differences (type-II). In the case, that the clones have notable (syntactical) differences, it can be difficult to remove them.

Third, we found FOP-related clones (type-II and type-III) that where not refactorable at all. In particular, one reason was that the application of refactorings implied complicated workarounds that outweigh the benefits of code clone removal. This observation lead us to the assumption that there is a border line where the extraction (of clones) is no longer beneficial for maintainability.

However, we are convinced that code clone removal is possible for a considerable fraction FOP-related clones and that refactorings are a viable approach to do this. Nevertheless, more research is necessary to find out reasons, characteristics and solutions for code clones and their removal in feature-oriented SPLs.

\section{Related Work}

The different fields of research addressed in this paper such as clone detection/removal or feature-oriented programming, has been subject to intensive research in the past. In this section, we discuss the relation to our work.

Many studies exist on code clones in object-oriented software systems. Some of them only focus on whether code clones exist or not $[6,10,31]$ whereas others analyze code clones with respect to their effects [32, 37], their removal [7] or other peculiarities, e.g., identifying crosscutting concerns [12]. However, all of these studies are limited to OOP (and, to a minor fraction, functional programming). By contrast, our work focuses on clone detection and analysis of particularities of FOP and SPLs, which has not been considered so far. We open a new field for code clone research activity. Additionally, we related the causes for FOP-related code 
clones to the limitations of FOP, which can initiate discussions on FOP language design.

Beyond FOP, clone detection in SPLs is rare as well. Mende et al. propose clone detection for supporting the evolution of SPLs [36]. However, in their work they consider SPLs, realized by object-oriented, preprocessor-based languages such as $\mathrm{C}++$ and thus, the individual features are separated only virtually, i.e., by syntactical elements such as \#ifdef. In our work, we consider feature-oriented SPLs where the features are separated into modules and we show that clone detection for such SPLs is applicable as well.

Because of the negative effects of code clones, their removal has been subject of research in the past. Balazinska et al. propose advanced code clone analysis that supports the application of objectoriented refactorings for code clone removal [7]. Higo et al. propose a metric-based approach to identify refactoring opportunities [20] for code clones. Although we focus on refactorings for code clone removal as well, our work is different in the way that we propose refactorings tailored to FOP which is in line aspect-oriented refactorings $[14,38]$. Specific details of such refactorings are out of the scope of this paper; for details see [33].

\section{Conclusion}

Code clones have negative effects on software systems. In this paper, we analyzed the existence of code clones in feature-oriented software product lines. To this end, we formulated research questions, regarding the causes and removal of code clones, specific to feature-oriented SPLs. Afterwards, we conducted an empirical analysis on ten different SPLs to answer these questions. Additionally, we performed a conceptual analysis on limitations of FOP and how these limitations contribute to code clones. Based on the results of the empirical analysis, we removed FOP-related code clones of an exemplary product line by the application of refactorings.

We observed, that code clones exist in feature-oriented product lines and that a considerable amount of these clones is FOP-related (in the context of SPLs). Furthermore, we found that these clones are caused by limitations of feature-oriented SPLs such as alternative features. Another interesting observation we made is that the development process is crucial for the amount of FOP-related code clones. More precisely, we found that feature-oriented SPLs, developed from scratch, contain a significantly higher amount of FOPrelated clones than SPLs decomposed from legacy applications. Finally, we observed that refactoring is a viable approach to remove FOP-related clones.

However, there still some questions we could not answer so far. More research is necessary to quantify which causes are crucial for FOP-related clones and which are not. Furthermore, it is open if clones are common to SPLs in general. For instance, in future work we will analyze how other mechanisms for dealing with variabilty such as \#ifdefs influence the occurence of code clones (compared to FOP). Nevertheless, our work and the results can serve as input for a discussion on code clones in feature-oriented SPLs and in SPL engineering in general.

\section{Acknowledgments}

We thank Thomas Thüm for giving insights to and the extension of FeatureIDE, which was invaluable for our work. Schulze's work has been funded in part by the German Federal Ministry of Education and Science(BMBF) through the Research Programme under Contract No. FKZ:13N10817. Apel's work is supported in part by DFG project \#AP 206/2-1. Kästner's work is supported in part by the European Union (ERC grant ScalPL \#203099).

\section{References}

[1] V. Alves and et al. Extracting and Evolving Mobile Games Product Lines. In Proc. of the Int. Software Product Line Conf., pages 70-81. Springer-Verlag, 2005.

[2] S. Apel. How AspectJ is Used: An Analysis of Eleven AspectJ Programs. Journal of Object Technology, 9(1):117-142, 2010.

[3] S. Apel, T. Leich, M. Rosenmüller, and G. Saake. FeatureC++: On the Symbiosis of Feature-Oriented and Aspect-Oriented Programming. In Proc. of the Int. Conf. on Generative Programming and Component Engineering, pages 125-140. Springer Verlag, 2005.

[4] S. Apel, T. Leich, and G. Saake. Aspectual Feature Modules. In IEEE Trans. Soft. Eng., volume 34, pages 162-180. IEEE Press, 2008.

[5] S. Apel, C. Kästner, and C. Lengauer. FeatureHouse: LanguageIndependent, Automated Software Composition. In Proc. Int. Conf. on Software Engineering, pages 221-231. IEEE Computer Society, 2009.

[6] B. S. Baker. On Finding Duplication and Near-Duplication in Large Software Systems. In Proc. of the Work. Conf. on Reverse Engineering, 1995.

[7] M. Balazinska et al. Advanced clone-analysis to support objectoriented system refactoring. In Proc. of the Work. Conf. on Reverse Engineering, 2000.

[8] H. Basit, D. Rajapakse, and S. Jarzabek. Beyond Templates: A Study of Clones in the STL and some General Implications. In Proc. Int. Conf. on Software Engineering, pages 451-459. ACM, 2005.

[9] D. Batory, J. Sarvela, and A. Rauschmayer. Scaling Step-Wise Refinement. IEEE Trans. Soft. Eng., 30:355-371, 2004.

[10] I. Baxter et al. Clone Detection Using Abstract Syntax Trees. In Proc. of the Int. Conf. on Software Maintenance, 1998.

[11] S. e. a. Bellon. Comparison and Evaluation of Clone Detection Tools. IEEE Trans. Soft. Eng., 33(9):577-591, 2007.

[12] M. Bruntink, A. Deursen, R. Engelen, and T. Tourw. On the Use of Clone Detection for Identifying Crosscutting Concern Code. In IEEE Trans. Soft. Eng., 2005.

[13] P. Clements and L. Northrop. Software Prodcut Lines: Practices and Patterns. Addison Wesley, 2006.

[14] P. Cole, L.and Borba. Deriving Refactorings for AspectJ. In Proc. Int. Conf. on Aspect-Oriented Software Development, pages 123-134. ACM, 2005.

[15] A. Colyer, R. A., and G. Blair. On the Separation of Concerns in Program Families. Technical Report Technical Report COMP-0012004, Computing Department, Lancaster University, 2004.

[16] E. Duala-Ekoko and M. Robillard. Tracking Code Clones in Evolving Software. In Proc. Int. Conf. on Software Engineering, 2007.

[17] S. Ducasse, M. Rieger, and S. Demeyer. A Language Independent Approach for Detecting Duplicated Code. In Proc. of the Int. Conf. on Software Maintenance, 1999.

[18] M. Fowler. Refactoring - Improving the Design of Existing Code. Addison Wesley, 2000

[19] Y. Higo, T. Kamiya, S. Kusumoto, and K. Inoue. Aries: Refactoring Support Environment based on Code Clone Analysis. In Proc. of Int'l Conf. on Soft. Eng. and Appl. (SEA), pages 222-229, 2004.

[20] Y. Higo, S. Kusumoto, and K. Inoue. A metric-based approach to identifying refactoring opportunities for merging code clones in a java software system. J. Softw. Maint. Evol., 20(6):435-461, 2008.

[21] L. Jiang, G. Misherghi, Z. Su, and S. Glondu. DECKARD: Scalable and Accurate Tree-based Detection of Code Clones. In Proc. Int. Conf. on Software Engineering, 2007.

[22] R. Johnson and B. Foote. Designing Reusable Classes. J. of ObjectOriented Progr., 1(2), 1988.

[23] E. Juergens, F. Deissenboeck, B. Hummel, and S. Wagner. Do Code Clones Matter? In Proc. Int. Conf. on Software Engineering, pages 485-495. IEEE Computer Society, 2009.

[24] T. Kamiya, S. Kusumoto, and K. Inoue. CCFinder: A Multilinguistic Token-Based Code Clone Detection System for Large Scale Source Code. In IEEE Trans. Soft. Eng., 2002. 
[25] K. Kang et al. Feature-Oriented Domain Analysis (FODA) Feasibility Study. Technical Report CMU/SEI-90-TR-21, Software Engineering Institute, Carnegie Mellon University, 1990.

[26] C. Kapser and M. W. Godfrey. Supporting the Analysis of Clones in Software Systems: A Case Study. J. Softw. Maint. Evol., 18(2):61-82, 2006.

[27] C. Kästner, S. Apel, and M. Kuhlemann. Granularity in Software Product Lines. In Proc. Int. Conf. on Software Engineering, pages 311-320. ACM, 2008.

[28] C. Kästner, S. Apel, and M. Kuhlemann. A Model of Refactoring Physically and Virtually Separated Features. In Proc. of the Int. Conf. on Generative Programming and Component Engineering, pages 157166. ACM, 2009.

[29] G. Kiczales et al. Aspect-oriented Programming. In Proc. of the Eur. Conf. on Object-Oriented Programming, 1997.

[30] K. Kontogiannis. Evaluation Experiments on the Detection of Programming Patterns Using Software Metrics. In Proc. of the Work. Conf. on Reverse Engineering, 1997.

[31] J. Krinke. Identifying Similar Code with Program Dependence Graphs. In Proc. of the Work. Conf. on Reverse Engineering, 2001.

[32] Z. Li, S. Lu, S. Myagmar, and Y. Zhou. CP-Miner: Finding CopyPaste and Related Bugs in Large-Scale Software Code. IEEE Trans. Soft. Eng., 32(3):176-192, 2006.

[33] J. Liu, D. Batory, and C. Lengauer. Feature Oriented Refactoring of Legacy Applications. In Proc. Int. Conf. on Software Engineering, pages 112-121. ACM, 2006.

[34] R. Lopez-Herrejon and D. Batory. A Standard Problem for Evaluating Product-Line Methodologies. In Proc. of the Int'l Conf. on Generative and Component-Based Soft. Eng. (GCSE), pages 10-24. SpringerVerlag, 2001.

[35] J. Mayrand, C. Leblanc, and E. Merlo. Experiment on the Automatic Detection of Function Clones in a Software System Using Metrics. In Proc. of the Int. Conf. on Software Maintenance, 1996.

[36] T. Mende, F. Beckwermert, R. Koschke, and G. Meier. Supporting the Grow-and-Prune Model in Software Product Lines Evolution Using Clone Detection. In Proc. of the Eur. Conf. on Software Maintenance and Reengineering, pages 163-172. IEEE Computer Society, 2008.

[37] A. Monden, D. Nakae, T. Kamiya, S. Sato, and K. Matsumoto. Software Quality Analysis by Code Clones in Industrial Legacy Software. In Proc. of the Int Symposium on Software Metrics, page 87. IEEE Computer Society, 2002.

[38] M. Monteiro. Catalogue of Refactorings for AspectJ. Technical report, Departamento de informtica, Escola de Engenharia, Universidada do Minho, 2004.

[39] G. Murphy, A. Lai, R. Walker, and M. Robillard. Separating Features in Source Code: An Exploratory Study. In Proc. Int. Conf. on Software Engineering, pages 275-284. IEEE Computer Society, 2001.

[40] D. L. Parnas. On the Criteria to be used in Decomposing Systems into Modules. Commun. ACM, 15(12):1053-1058, 1972.

[41] P. W. Pearse, T. T.and Oman. Experiences Developing and Maintaining Software in a Multi-Platform Environment. In Proc. of the Int. Conf. on Software Maintenance, pages 270-277. IEEE Computer Society, 1997.

[42] C. Prehofer. Feature-Oriented Programming: A Fresh Look at Objects. In Proc. of the Eur. Conf. on Object-Oriented Programming, pages 419-443. Springer, 1997.

[43] C. Roy and J. Cordy. A Survey on Software Clone Detection Research. Technical Report 2007-451, School of Computing, Queen's University at Kingston, 2007.

[44] C. K. Roy, J. Cordy, and R. Koschke. Comparison and Evaluation of Code Clone Detection Techniques and Tools: A qualitative Approach. Sci. Comp. Prog., 74(7):470-495, 2009.

[45] T. Thüm, D. Batory, and C. Kästner. Reasoning about edits to feature models. In Proc. Int. Conf. on Software Engineering, pages 254-264. IEEE Computer Society, 2009.
[46] M. Toomim, A. Begel, and S. Graham. Managing Duplicated Code with Linked Editing. In Proc. of the Symposium on Visual Languages - Human Centric Computing, pages 173-180. IEEE Computer Society, 2004. 